\title{
Modeling of the Marine atmosphere and its impact on Ka-band channels
}

\author{
Zheng Liu*, Fu-an Sun, and Bin Zhou \\ China Satellite Maritime Tracking and Controlling Department, Jiangyin 214431, China
}

\begin{abstract}
The sea atmosphere environment will affect the Ka frequency channel in TT\&C. Firstly, this paper briefly introduces the Marine atmospheric environment. Attenuation models of water vapor solubility and rainfall intensity are established. The variation characteristics of atmospheric environment and the estimation method of rainfall intensity are studied. Finally, the influence of Marine atmosphere on Ka-band channel is simulated and analyzed. The simulation results show that different elevation angles have different effects on Ka-band channels. The influence result decreases gradually with the elevation Angle increasing.
\end{abstract}

Keywords: Atmospheric environment, Ka-band, Channel.

\section{Introduction}

Maritime space refers to the electromagnetic waves emitted by offshore test stations that travel through the atmosphere. The range is $150 \mathrm{~km}$ below sea level. The complex changes of atmospheric environment, temperature, density, air pressure and meteorological state directly affect the characteristics of the channel as the electromagnetic wave passes through the atmosphere. The channel characteristic changes mainly include attenuation and phase changes. The higher the frequency band, the greater the attenuation, the more unstable the signal polarity and the greater the change of phase value. It will seriously affect the Angle tracking accuracy and even the target loss state, which will lead to the communication attenuation to the spacecraft. Therefore, it is very significant to study the influence of Marine atmospheric environment and Ka-band channel to help the reasonable planning of measurement and control scheme.

\section{Modeling of the Marine atmospheric environment}

\subsection{Marine atmosphere}

The parameters of the Marine atmosphere environment can describe the physical quantity of the state and change of the Marine atmosphere. Its basic parameters include temperature, humidity, air pressure, rainfall intensity, number of clouds, distance, pitch Angle and so on.

*Corresponding author: liuzheng354@qq.com 
The sea atmosphere includes rain clouds, the troposphere and the ionosphere. The temperature decreases as the height increases. Humidity also decreases with height. When the altitude reaches $5 \mathrm{~km}$, the temperature approaches $0^{\circ} \mathrm{C}$. When the height of the cloud layer reaches $20 \mathrm{~km}$, the air is extremely thin, the environmental water content is low and there is no rain or snow. When it get to an altitude of 80 kilometers, the atmosphere starts to ionize because of ultraviolet light from the sun. The air contains electrons, ions, and highenergy protons and neutrons, including those from outer space.Ka band signals are greatly affected by rain decline, atmospheric propagation loss, polarization and noise. In order to simplify the estimation formula, meteorological attenuation is divided into rain decline, atmospheric attenuation and noise attenuation. The basic formula is shown in Formula.

$$
A=A_{r}+A_{q}+A_{t}
$$

where, $A$ is total attenuation, $A_{q}$ is atmospheric attenuation, $A_{r}$ rain attenuation, $A_{t}$ is noise attenuation.

\subsection{Atmospheric attenuation model}

Energy loss is one of the main effects of the atmosphere on microwave propagation. Because of the absorption and scattering of oxygen molecules and water vapor molecules in the troposphere, and the absorption of free electrons and ions in the ionosphere, microwave propagation in the atmosphere will cause energy loss.

$$
A_{q}=h \gamma
$$

where, $h$ is the equivalent length $(\mathrm{km})$ of radio waves travelling through the atmosphere, $\gamma$ is the equivalent attenuation coefficient $(\mathrm{dB} / \mathrm{km})$. The gases in the atmosphere that absorb more electromagnetic waves are mainly oxygen and water vapor. The attenuation coefficient of atmospheric loss is therefore related to humidity and temperature. The basic formula is as follows:

$$
\gamma=a+b \rho_{0}+c T_{0}
$$

where, the coefficients $\mathrm{a}, \mathrm{b}$ and $\mathrm{c}$ are related to frequency; $\rho_{0}$ is the average local surface water vapor concentration $(\mathrm{g} / \mathrm{m} 2)$, which is related to humidity $(\%) ; T_{0}$ is the average (OC) of the local surface.

$h_{s}$ is the actual length of travel through the atmosphere. It is estimated according to the target distance, pitch Angle change. When the target is in the atmosphere, $\mathrm{h}$ is equal to the measured distance $\mathrm{R}$. When the target is outside the atmosphere, $\mathrm{h}$ is related to the altitude of the atmosphere, $\mathrm{h}_{\mathrm{q}}$, the elevation of the antenna, $\mathrm{E}$, and the altitude of the antenna, $\mathrm{h}_{0}$. The intensity of atmospheric attenuation varies dynamically with height. The equivalent altitude is divided into two parts according to $20 \mathrm{~km}$. The part above $20 \mathrm{~km}$ is evaluated.

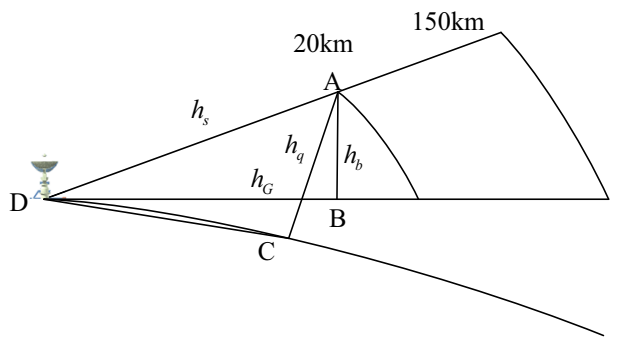

Fig. 2. Schematic diagram of atmospheric attenuation distance. 
The actual length of the wave through the atmosphere $h_{s}$ is $A D$. It has to do with the relative antenna height of the atmosphere being $h_{b}(\mathrm{AB})$ and the elevation Angle $\mathrm{E}(\angle$ $\mathrm{ADB}$ ), as shown in Figure 2,

$$
h_{s}=h_{b} / \sin E
$$

However, the atmospheric height of point $A$ is actually $h_{q}(A C)$, which is larger than the relative antenna height $h_{b}(\mathrm{AB})$. The actual height is calculated as follows:

$$
h_{l}=h_{q} / \sin E
$$

It means that the actual length of the atmosphere that the wave travels through is less than HL, which means that it has to be corrected in the direction of decreasing H.It is multiplied by a shortening factor, sp, which can be estimated as follows

$$
s_{q}=1 /\left(1+\cos E \times h_{q} / \sin E /\left(\sqrt{\left(R+h_{q}\right)^{2}-R^{2}}\right)\right)
$$

where $\mathrm{R}$ is the radius of the earth.The calculation formula of equivalent height $\mathrm{H}$ is as follows,

$$
h=s_{q} \times h_{l}
$$

\subsection{Rain attenuation model}

Raindrops absorb and scatter radio waves causing them to decay as they travel through rain-falling areas. The radius of raindrops is generally between $0.025 \mathrm{~cm}$ and $0.3 \mathrm{~cm}$. The wavelength of $\mathrm{Ka}$ operating frequency band is around $1 \mathrm{~cm}$. Its rain decline is mainly absorption attenuation and mostly heat loss. Because rainfall varies from region to region and season to season. Its influence is uneven in time and space. Therefore, their system characteristics must be measured over a long period of time. And each site often do not have this condition. Therefore, meteorological data such as rainfall amount and probability of rainfall time are used to predict rainfall attenuation value. At present, this paper is expected to use ITU-R method to simplify the calculation method, which requires fewer parameters and higher accuracy. The relationship between rainfall intensity $R_{p}(\mathrm{~mm} / \mathrm{h})$ and rainfall attenuation $A_{R}(\mathrm{~dB} / \mathrm{km})$ is expressed as follows:

$$
A_{R}=d R_{p}^{e} L_{e}
$$

where $L_{e}$ is the effective path length of the radio wave through the rain region. $d$ and e are the coefficients of frequency and temperature.

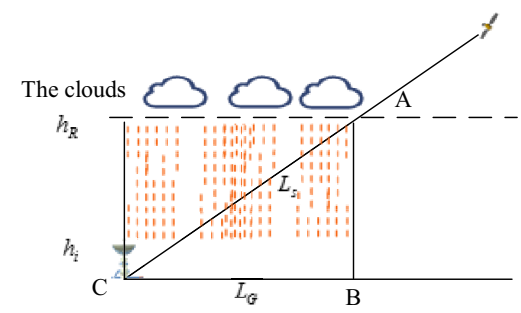

Fig. 3. Schematic diagram of equivalent path of rain attenuation.

The calculation formula of LE is as follows,

$$
L_{e}=\gamma_{p} \times L_{s}
$$


$\gamma_{p}$ is the shortening factor, and $L_{s}$ (AC) is the actual length of the radio wave through the rain region. It is related to the height of rainfall layer $h_{R}(\mathrm{AB})$, the height of antenna $h_{i}$, and the elevation Angle of antenna $\mathrm{E}(\angle \mathrm{ACB})$.

$$
L_{s}=\left(h_{R}-h_{i}\right) / \sin E
$$

In the formula, $h_{i}$ is the altitude of the antenna. $h_{R}$ is the height of the rain layer. Equation (10) represents the raindrops within the height range of $\left(h_{R}-h_{i}\right)$. $L_{G}=L_{s} \cos \theta$ also raining on the ground. The actual range of ground rain area is less than this value. The actual rain area length through which the wave passes is less than $L_{s}$ and needs to be corrected in the direction of decreasing $L_{e}$.It can be estimated according to the following formula.

$$
\gamma_{p}=1 /\left(1+L_{G} / L_{0}\right)
$$

In the formula, $\gamma_{p}$ is the shortening factor, and $L_{0}$ is the ground coverage of normal rain area.

$$
L_{0}=35 \exp \left(-0.015 R_{p}\right)
$$

\subsection{Noise attenuation model}

Rain attenuation and atmospheric attenuation not only affect the generation of channel links. Its effect on the antenna is similar to that of a lossy waveguide. Under different meteorological conditions, the noise temperature of the antenna will have an effect. The increase of system noise temperature $A_{R}$ caused by rain decline $A_{q}$ and atmospheric attenuation $\Delta T_{A}$ can be calculated by:

$$
\Delta T_{A}=T_{r}\left(1-\frac{1}{A_{R}+A_{q}}\right)
$$

where $T_{r}$ is the antenna temperature, which is usually taken as $273 . A_{R}$ is rain decline, $A_{q}$ is atmospheric attenuation, and they are expressed in multiples. The change of noise temperature needs to be influenced by the equivalent noise temperature $\mathrm{T}$ of the equipment.

\section{Identification of key parameters in atmospheric environment model}

The atmospheric environment model involves temperature, humidity, pressure, rainfall intensity and distance parameters. The characteristics of tracking meteorological attenuation within the arc can be responded to improve the practical application of the model. The parameters of the weather and the order of the formula can be controlled to establish the relationship between the characteristic variables and the internal variables of the formula, which finds the influence factors of the channel characteristics. This paper attempts to use numerical analysis method and empirical formula method to identify the parameters of atmospheric environment model. The experimental data are used as the basis to obtain the measurable data of the relevant parameters of the model. 


\subsection{Parameter identification of atmospheric attenuation model}

In the atmospheric attenuation model, $\rho_{0}$ is the average concentration of local surface water vapor $(\mathrm{g} / \mathrm{m} 2)$, which is related to humidity and pressure. It establishes a formula for estimating $\rho_{0}$.

$$
\rho_{0}=a h+b
$$

The average concentration of water vapor under different humidity conditions is tested, When the humidity is below 9.98 percent, the average concentration of water vapor is about 0 .

Through analysis, the attenuation coefficient $\gamma$ in the atmospheric attenuation formula is used to identify the coefficients $a, b$ and $c$ in the state of $\rho_{0}$ and $T_{0}$. The attenuation coefficients of known typical frequencies are shown in Table 1.

Table 1. Attenuation coefficient at different frequencies.

\begin{tabular}{cccc}
\hline Frequency $/ \mathrm{GHz}$ & $\mathrm{a}$ & $\mathrm{b}$ & $\mathrm{C}$ \\
\hline 24 & 0.00823 & 0.00090 & 0.0000890 \\
30 & 0.00825 & 0.00110 & 0.0000899 \\
35 & 0.00827 & 0.0013 & 0.0000904 \\
\hline
\end{tabular}

The logarithmic scale insertion method is used to obtain the corresponding parameters of the required frequency. The formula is as follows:

$$
x(f)=\left(x_{2}-x_{1}\right) \bullet\left[\frac{\lg \left(f / f_{1}\right)}{\lg \left(f_{2} / f_{1}\right)}\right]+x 1
$$

\subsection{Parameter identification of rain attenuation model}

$\mathrm{d}$ and e are the frequency dependent coefficients in the rain attenuation model. Its typical frequency values are shown in Table 2.

Table 2. Variation of $a$ and $b$ with frequency in the rain attenuation formula.

\begin{tabular}{ccc}
\hline Frequency/GHz & $\mathrm{d}$ & $\mathrm{e}$ \\
\hline 20 & 0.0671 & 1.065 \\
30 & 0.087 & 1 \\
40 & 0.31 & 0.929 \\
\hline
\end{tabular}

The logarithmic scale insertion method is used to determine the desired frequency parameters.

The Meteorological Society obtains rainfall levels from weather forecasts. The International Meteorological Association publishes data based on the probability of rainfall in each region. In the rain-decline model, $h_{R}$ is the height of rain layer. The Meteorological Society gives an isotherm height of $0^{\circ} \mathrm{C}$.

\section{Simulation and characteristic analysis of sea atmosphere environment}

In order to verify the practicability of the model, parameters of the Marine atmosphere environment were set into the model to estimate the simulation attenuation. T\&C scenarios are configured in conjunction with meteorological associations to provide Marine data and key parameters. Atmospheric Attenuation Model: Parameters in the atmospheric 
attenuation model are different with different data of temperature, humidity and pressure. the results are shown in Fig 3:

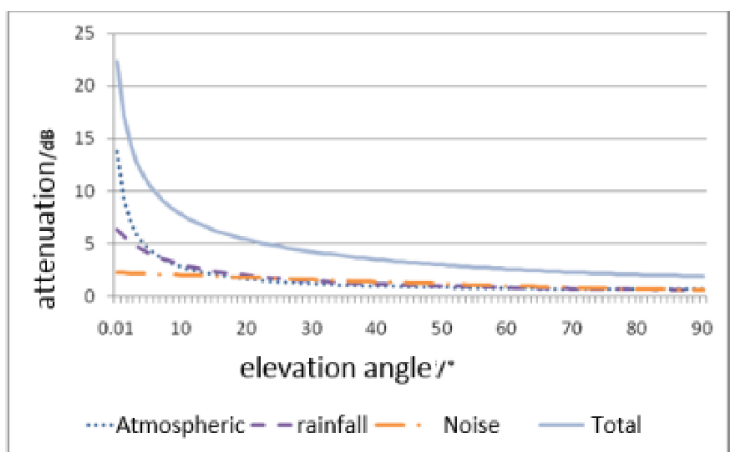

Fig. 3. Curve of attenuation of Ka frequency channel by the Marine atmosphere environment.

As can be seen from Fig. 3, the Marine atmosphere has a great influence on channel attenuation in the Ka band. When it is low elevation, the attenuation increases exponentially. When the pitch Angle is raised, the attenuation tends to be stable.

\section{Summary}

In this paper, the channel attenuation under the condition of Marine atmosphere environment is introduced, and the influence factors such as temperature, humidity, rainfall, and the change of pitch Angle are analyzed. The simulation analysis in this paper shows that the signal in Ka frequency band is greatly affected by the Marine atmospheric environment when the target is in and out of the horizon and in the state of low elevation. It decreases as the pitch Angle increases. Therefore, the influence of the Marine atmosphere environment on the channel is fully considered, which can improve the reliability of the channel link in the subsequent design of measurement and control link.

\section{References}

1. Ojo, J.S., (2008). Rain rate and rain attenuation prediction for satellite communication in ku and ka bands over nigeria. ,5,(2008), 03, 207-223

2. Mandeep, J. S.(2007). Rain attenuation predictions at ku-band in south east asia countries. ,76,(2007), 76, 65-74.

3. (2021). Second order experimental statistics of rain attenuation at ka band in a tropical location. Advances in Space Research 